\title{
Softwares para repositorios digitales: evaluación para la gestión y preservación de los materiales bibliográficos y de los documentos archivísticos de la Biblioteca Nacional de Brasil
}

\author{
Wellington da Silva \\ Biblioteca Nacional, Rio de Janeiro, RJ, Brasil \\ wellington.silva@bn.gov.br \\ José Antonio Merlo Vega \\ Universidad de Salamanca, Depto. Biblioteconomía y Documentación, Salamanca, España \\ merlo@usal.es
}

DOI: https://doi.org/10.26512/rici.v13.n1.2020.29554

Recebido/Recibido/Received: 2019-12-15

Aceitado/Aceptado/Accepted: 2020-02-09

Resumen: La llegada de la Tecnología de la Información y Comunicación cambió la manera de la Biblioteca Nacional de Brasil de producir, usar y almacenar información, si antes su acervo estaba compuesto de pergaminos, papiros, libros y documentos administrativos en soporte papel, actualmente se compone además por objetos digitales (nativos y digitalizados) como libros, revistas, periódicos, música, fotografías, documentos administrativos entre otros; y el volumen de estos objetos digitales están creciendo cada día. Por eso la investigación evaluó y analizó las características y las funcionalidades de los softwares para repositorios digitales Archivematica, DSpace y RODA con el fin de identificar el más adecuado para la gestión y preservación de los materiales bibliográficos y de los documentos archivísticos de la Biblioteca Nacional de Brasil. Se revisó la estructura organizacional de la Biblioteca Nacional de Brasil y la bibliografía especializada; se analizó los softwares seleccionados mediante un criterio cuantitativo basado en las funcionalidades que cumplen. DSpace fue el que presentó mejor resultado, cumple con el $92 \%$ de las funcionalidades seleccionadas. En segundo lugar está RODA, con un nivel de satisfacción del $77 \%$ y en último lugar está Archivematica, con $62 \%$. Archivematica no es un software para repositorio digital, sino un sistema de preservación digital, es decir, una solución tecnológica para un repositorio digital. RODA, es un software de preservación OAIS y no una plataforma de publicación. DSpace es el software más adecuado para la gestión y preservación de los materiales bibliográficos y de los documentos archivísticos de la Biblioteca Nacional de Brasil, atendiendo al $92 \%$ de las funcionalidades consideradas como las más importantes y relevantes para la gestión y preservación.

Palabras clave: preservación digital. repositorio digital. evaluación. biblioteca nacional. Brasil.

Software for digital repositories: evaluation for the management and preservation of bibliographic materials and archival documents of the National Library of Brazil

abstract: The Information and Communication Technology changed the way of the National Library of Brazil to produce, use and store information, if before its collection was composed of palimpsest, papyrus, books and administrative documents on paper support, it is currently also composed for digital objects (native and digitized) such as books, magazines, newspapers, music, photographs, administrative documents among others; and the number of these digital objects are growing every day. Therefore, the research evaluated and analyzed the characteristics and functionalities of the software for digital repositories Archivematica, DSpace and RODA in order to identify the most suitable for the management and preservation of bibliographic materials and archival documents of the National Library of Brazil. The organizational structure of the National Library of Brazil and the specialized bibliography 
were reviewed; The software selected were analyzed using a quantitative criterion based on the functionalities they fulfill. DSpace was the one that presented the best result, fulfills $92 \%$ of the selected functionalities. In second place is RODA, with a level of satisfaction of $77 \%$ and lastly is Archivematica, with $62 \%$. Archivematica is not a digital repository software, but a digital preservation system, that is, a technological solution for a digital repository. RODA, is an OAIS preservation software and not a publishing platform. DSpace is the most suitable software for the management and preservation of bibliographic materials and archival documents of the National Library of Brazil, serving $92 \%$ of the functionalities considered as the most important and relevant for management and preservation.

Keywords: digital preservation. digital repository. evaluation. national library. Brazil.

\section{Programas para repositórios digitais: avaliação para o gerenciamento e preservação de materiais bibliográficos e documentos de arquivo da Biblioteca Nacional do Brasil}

Resumo: A chegada da Tecnologia da Informação e Comunicação mudou a maneira da Biblioteca Nacional do Brasil de produzir, usar e armazenar informações, se antes sua coleção era composta por pergaminhos, papiros, livros e documentos administrativos em suporte em papel, atualmente ela também é composta por objetos digitais (nativos e digitalizados), como livros, revistas, jornais, músicas, fotografias, documentos administrativos, entre outros; e o volume desses objetos digitais está aumentando a cada dia. Por isso, a pesquisa avaliou e analisou as características e funcionalidades dos softwares para repositórios digitais Archivematica, DSpace e RODA, a fim de identificar os mais adequados para gestão e preservação de materiais bibliográficos e documentos de arquivo da Biblioteca Nacional de Brasil. A estrutura organizacional da Biblioteca Nacional do Brasil e a bibliografia especializada foram revisadas; os softwares selecionados foram analisados utilizando um critério quantitativo com base nas funcionalidades que eles cumprem. DSpace foi o que apresentou o melhor resultado, cumpre $92 \%$ das funcionalidades selecionadas. Em segundo lugar, está o RODA, com um nível de satisfação de $77 \%$ e, por último, zo Archivematica, com 62\%. $\theta$ Archivematica não é um software de repositório digital, mas um sistema de preservação digital, ou seja, uma solução tecnológica para um repositório digital. RODA, é um software de preservação OAIS e não uma plataforma de publicação. $O$ DSpace é o software mais adequado para gestão e preservação de materiais bibliográficos e documentos de arquivo da Biblioteca Nacional do Brasil, pois atende a $92 \%$ das funcionalidades consideradas como as mais importantes e relevantes para gestão e preservação.

Palavras chave: preservação digital. repositório digital. avaliação. Biblioteca Nacional. Brasil.

\section{Introducción}

Actualmente, la mayoría de las instituciones, utilizan las funcionalidades ofrecidas por la tecnología de la información y comunicación (TIC) para producir, gestionar, almacenar y difundir la información. Desde el escenario globalizado de la Administración Pública, se ha adoptado procesos de modernización, que buscan mejorar la calidad de los servicios públicos y aproximar al ciudadano de la administración (MATEUS, 2008). Lo que comprueba la existencia de un proceso de modernización administrativa basado en la desmaterialización de la información. Para Thurston (2012), los gobiernos adoptan sistemas digitales sin tener conciencia de los desafíos que plantea preservar la integridad de los documentos generados por tales sistemas. Estar consciente de eso significa reflexionar sobre las consecuencias del uso de esas nuevas tecnologías, pues estas consecuencias pueden dificultar o imposibilitar el acceso y la comprensión de los objetos digitales en el futuro, debido a la obsolescencia tecnológica (hardware y software), la fragilidad de los soportes y también por problemas para asegurar la autenticidad y la integridad de la información registradas en los soportes y 
formatos digitales. Según Álvarez Wong (2017), la consecuencia de estos problemas puede ser una pérdida de información con daños irreparables para la gestión y memoria de una institución y de la sociedad.

La longevidad de los objetos digitales depende directamente del medio de almacenamiento usado Texier (2013). Por esto uno de los principales enfoques de la preservación digital es el almacenamiento de la información. De acuerdo con Heminger y Robertson (2000), esto se debe a que las nuevas tecnologías de producción y almacenamiento de objetos digitales están evolucionando más rápido que nunca y la rapidez del desarrollo exige la preservación del gran volumen de conocimiento que se ha creado y se sigue creando en el entorno digital. Para que los objetos digitales sean preservados y almacenados con seguridad y control se debe tener en cuenta la implantación de un Repositorio Digital. PolancoCortés (2014) define Repositorio Digital, en un sentido amplio, como un medio para gestionar, almacenar, preservar, difundir y facilitar el acceso a los objetos digitales que almacena.

La Biblioteca Nacional de Brasil (BNB) también cambió su manera de producir, usar y almacenar información, debido a las TIC. Si antes su acervo estaba compuesto de pergaminos, papiros, libros y documentos administrativos en soporte papel, actualmente se compone además por objetos digitales (nativos y digitalizados) como libros, revistas, periódicos, música, fotografías, documentos administrativos entre otros; y el volumen de estos objetos digitales están creciendo cada día.

Con el objetivo de ampliar dos de sus principales competencias, o sea, preservar la memoria cultural y proporcionar el amplio acceso a las informaciones contenidas en su acervo, en 2000 la BNB empezó con el proyecto de digitalización de sus colecciones bibliográficas, proyecto que originó la Biblioteca Nacional Digital (BNDigital). La BNDigital tiene la misión de ser un vehículo diseminador de la memoria cultural brasileña en todo el país y en el exterior, y aumentar el contenido en portugués disponible en la web, entre otros.

Además de las colecciones bibliográficas el acervo de la BNB también está compuesto por el fondo archivístico, que son documentos producidos en el transcurso de las actividades administrativas, cualquiera que sea el soporte, (CONSELHO NACIONAL DE ARQUIVOS, 2011). Los documentos archivísticos tienen un alto valor administrativo y legal para la institución, principalmente porque la BNB es una institución pública y estos documentos dan soporte a la democracia, protegen y mantienen derechos de los ciudadanos, y convierten las acciones institucionales en transparentes, constituyendo un importante instrumento contra la corrupción.

La creación de la BNDigital y la implantación del SEI cambiaron principalmente el uso y el almacenamiento de las colecciones bibliográficas digitalizadas y del fondo archivístico digital 
(nativo). Antes de la creación de la BNDigital los documentos estaban, en su gran mayoría, en soporte papel y almacenados en los almacenes de la institución. Hasta la implantación del SEI, los documentos archivísticos eran producidos en el entorno digital, y aquellos con valor administrativo y legal, eran migrados para soporte papel, es decir, su producción era digital, pero su uso, almacenamiento y la gestión eran realizados por medios analógicos.

Considerando que la llegada de las TIC afectó la rutina diaria de la BNB, posibilitó ampliar la difusión de sus acervos, que es parte de su misión, mejoró la productividad y el rendimiento de sus actividades. Lo Decreto n. 8.297/2014 establece en el artículo 2o que la BNB debe adoptar medidas necesarias para la preservación y protección del patrimonio bibliográfico y digital bajo su custodia, y del Decreto n. 8.539/2015 establecen el artículo 18 que los órganos o entidades deberán establecer políticas, estrategias y acciones que garanticen la preservación, el acceso y el uso continuo a largo plazo de los documentos digitales. Por eso la Biblioteca Nacional de Brasil tendrá que adoptar nuevas estrategias para la preservación y protección de sus colecciones bibliográficas digitalizadas y del fondo archivístico digital (nativo), para cumplir con las obligaciones establecidas en los dispositivos legales mencionados. Una de las estrategias que la BNB puede adoptar para la preservación digital de sus acervos es la implantación de un repositorio digital, que sea adecuado a la gestión y preservación de los materiales bibliográficos y de los documentos archivísticos.

Actualmente hay una gran variedad de software para repositorios digitales disponible en el mercado, por eso es necesario evaluar algunos para identificar el más adecuado a las necesidades de la BNB. Basado en esta necesidad, el objetivo de esta investigación es evaluar y analizar las características y las funcionalidades de los softwares Archivematica, DSpace y RODA, con el fin de identificar el más adecuado a la gestión y preservación de los materiales bibliográficos y de los documentos archivísticos. Los objetos digitales (digitalizados) que forman la BNDigital y los documentos archivísticos digitales (nativos) producidos por el Sistema Eletrônico de Informações (SEI) es el alcance de la presente investigación.

\section{Metodología}

La metodología utilizada para realizar esta investigación se desarrolló en diferentes etapas: en la primera etapa realizó una revisión de la estructura organizacional de la Biblioteca Nacional de Brasil. Este estudio se restringió a los documentos constitutivos de la institución, es decir, los documentos legales donde están definidos su misión, sus objetivos, competencias y actividades. La finalidad del estudio fue identificar en la legislación las obligaciones legales con respecto a la preservación y difusión, que podrán refrendar los objetivos propuestos en este trabajo. La segunda etapa fue una revisión de la bibliografía especializada, esta etapa 
consistió en seleccionar artículos relevantes, centrados en evaluación de las características y funcionalidades de los softwares para repositorios digitales, publicados a partir del año 2008. De la bibliografía especializada revisada, los trabajos que se revelaron como más relevantes y adecuados para lograr el objetivo de esta investigación fueron:

- Evaluation of Three Open Source Software in Terms of Managing Repositories of Electronic Theses and Dissertations: A Comparison Study (Masrek y Hakimjavadi 2012): evalúa las características de tres software de Código abierto (DSpace, EPrints, Fedora).

- Open Source Software for Digital Preservation Repositories: A Survey (Rosa, Craveiros y Domingues, 2017):se centra en el estado del arte de once soluciones de software de código abierto para el campo de la preservación digital que se utiliza para asimilar y difundir información.

- Diretrizes para a Implementação de Repositórios Digitais Confiáveis de documentos arquivísticos. Conselho Nacional de Arquivos (2014): indica requisitos para repositorios confiables de documentos archivísticos digitales, para garantizar la integridad, la autenticidad, la confidencialidad, la disponibilidad, el acceso y la preservación, teniendo en cuenta la perspectiva de la necesidad de mantener los acervos documentales a largo plazo o, incluso, permanentemente.

- Institutional Repository Software Comparison Bankier y Gleason (2014): compara las características de los principales software y está destinada a ayudar a las bibliotecas a centrarse en qué características ayudarán a facilitar el éxito de su repositorio.

Para elegir los softwares a ser evaluados en esta investigación, fueron consideradas diferentes características y funcionalidades como: el tipo de licencia, es decir, que debería ser libre y de código abierto, el porcentaje de instalaciones registradas en el mundo, incluso en Brasil y los tipos de instituciones que utilizaban el software, debido a que la presente investigación está centrada en materiales bibliográficos y archivísticos, es decir se valoró detenidamente los softwares implantados en bibliotecas y archivos. Los softwares que atendían mejor a los requisitos establecidos fueron: Archivematica, DSpace y RODA.

Después de elegir los softwares, fueron seleccionadas las funcionalidades más importantes y relevantes a ser evaluadas. Teniendo en cuenta el objetivo de la presente investigación y las necesidades de la Biblioteca Nacional de Brasil, las características consideradas más importantes y relevantes fueron: complejidad de instalación, usabilidad, flexibilidad y escalabilidad. A partir de dichas características fueron definidas seis categorías: Infraestructura, Gestión de contenidos, Administración del uso, Interfaz de usuario, 
Preservación digital e Interoperabilidad e Integración. Dentro de cada categoría fueron elegidas las funcionalidades más utilizadas en la bibliografía consultada, totalizando 40 de ellas. Para esta elección se consideró: los requisitos técnicos para la instalación, la seguridad de la información, estrategias de preservación, interfaz de usuario e integración. A continuación se describe las funcionalidades de cada categoría:

- Infraestructura: Facilidad para instalar, Facilidad para actualizar, Código fuente modificable, Documentación disponible, Soporta Windows, Soporta Linux, Soporta Java, Plataforma para publicación web, Flexibilidad para personalización, Capacidad de memoria y procesador bajo, Numerosa comunidad para soporte, Soporte externo en Brasil o en portugués y Madurez en el mercado.

- Gestión de contenido: Restricción de acceso a información (pública/privada), Flujo de trabajo (workflow), Acepta múltiples formatos, Búsqueda avanzada/facetada, Búsqueda por texto integral, Opción de compartir en redes sociales.

- Administración del uso: Autenticación, Diferentes permisos de acceso, Registro de acciones de usuario, Notificaciones y Estadísticas.

- Interfaz de usuario: Personalización, Acceso multilingüe en la interfaz, Administración multilingüe y Adaptación para móvil.

- Preservación digital: Modelo OAIS, PREMIS, METS, EAD, Dublin Core, Exportar a/Importar desde otro formatos de metadatos; Herramientas/servicios de conversión de formatos, Validación e Identificación de formatos y Respaldo/Copia de Seguridad.

- Interoperabilidad e Integración: OIA-PMH, Integración con otros sistemas e Integración con los softwares evaluados.

Definidos los softwares y las funcionalidades a ser evaluadas, fue necesario definir cuál metodología de evaluación sería utilizada para alcanzar el resultado. Para esta etapa se utilizó el modelo creado por Alvite y Rodríguez (2002), para evaluar las interfaces de OPACs implementadas con UNICORN en las Universidades madrileñas. Para este trabajo se consideró solamente la manera como las autoras puntuaban cada indicador, que consistía en atribuir 0 (ausencia), 1 (aplicación incompleta/limitada) y 2 (aplicación completa). Es decir, cada funcionalidad seleccionada se puntuó con 0,1 o 2, en que 0 significa que el software no posee la funcionalidad, 1 que la posee de manera incompleta o limitada y 2 completa.

\section{Resultados}

En este apartado se presentan los resultados obtenidos por los softwares en cada categoría y en general, originados a partir de la suma de los puntos atribuidos a cada 
funcionalidad. Se sumó la puntuación obtenida en cada categoría, así como la puntuación máxima que cada software podría obtener. Estas puntuaciones se convirtieron en un porcentaje, con el fin de representar el nivel de asistencia del software en cada categoría y en general.

\section{Resultados por categorías}

Categoría Infraestructura

\begin{tabular}{|l|c|c|c|}
\hline \multirow{2}{*}{\multicolumn{1}{|c|}{ Funcionalidades }} & \multicolumn{3}{c|}{ Puntuación } \\
\cline { 2 - 4 } & Archivematica & DSpace & RODA \\
& 1.9 .1 & $6 . x$ & 3.0 \\
\hline Facilidad para instalar & 0 & 2 & 2 \\
\hline Facilidad para actualizar & 0 & 1 & 1 \\
\hline Código fuente modificable & 2 & 2 & 2 \\
\hline Documentación disponible & 2 & 2 & 1 \\
\hline Soporta Windows & 1 & 2 & 2 \\
\hline Soporta Linux & 2 & 2 & 2 \\
\hline Soporta Java & 0 & 2 & 2 \\
\hline Plataforma para publicación web & 0 & 2 & 0 \\
\hline Flexibilidad para personalización & 2 & 2 & 2 \\
\hline Capacidad de memoria y procesador bajo & 0 & 1 & 0 \\
\hline Numerosa comunidad para soporte & 1 & 2 & 0 \\
\hline Soporte externo en Brasil o en portugués & 2 & 2 & 1 \\
\hline Madurez en el mercado & 1 & 2 & 0 \\
\hline \multicolumn{1}{|c|}{ suBTOTAL } & $13 / 26$ & $24 / 26$ & $15 / 26$ \\
\hline
\end{tabular}

Tabla 1. Funcionalidades relacionadas con la infraestructura

De las trece funcionalidades, las que más sobresalen teniendo en cuenta la viabilidad de la implantación son la facilidad para instalar, la numerosa comunidad para soporte y madurez en el mercado. Pues al tener estas funcionalidades el software demuestra practicidad en su instalación, lo que se traduce en un coste más bajo para la implantación y también demuestran la satisfacción de los usuarios, por la numerosa comunidad de soporte y madurez en el mercado.

\section{Categoría Gestión de contenido}

\begin{tabular}{|l|c|c|c|}
\hline \multicolumn{1}{|c|}{ Funcionalidades } & \multicolumn{2}{c|}{ Puntación } \\
\cline { 2 - 4 } & Archivematica & $\begin{array}{c}\text { DSpace } \\
6 . x\end{array}$ & $\begin{array}{c}\text { RODA } \\
3.0\end{array}$ \\
\hline Restricción de acceso a información (pública/privada) & 1.9 .1 & 2 & 2 \\
\hline Flujo de trabajo (workflow) & 0 & 2 & 2 \\
\hline Acepta múltiples formatos & 2 & 2 & 2 \\
\hline Búsqueda Avanzada/facetada & 2 & 2 & 2 \\
\hline Búsqueda por texto integral & 2 & 2 & 2 \\
\hline Opción de compartir en redes sociales & 0 & 2 & 0 \\
\hline \multicolumn{1}{|c|}{ SUBTOTAL } & 0 & $\mathbf{6 / 1 2}$ & $\mathbf{1 2 / 1 2}$ \\
\hline
\end{tabular}

Tabla 2. Funcionalidades relacionadas con la Gestión de contenido 
Para gestionar los materiales bibliográficos (acceso abierto) y los documentos archivísticos (acceso bajo demanda) de la Biblioteca Nacional de Brasil es esencial que el software tenga la funcionalidad de restricción de acceso a información (pública/privada). No poseer esta funcionalidad es un punto débil que puede inhabilitar el software para ser el más adecuado.

Categoría Administración del uso

\begin{tabular}{|c|c|c|c|}
\hline \multirow[b]{2}{*}{ Funcionalidades } & \multicolumn{3}{|c|}{ Puntuación } \\
\hline & $\begin{array}{c}\text { Archivematica } \\
\text { 1.9.1 } \\
\end{array}$ & $\begin{array}{c}\text { DSpace } \\
6 . x \\
\end{array}$ & $\begin{array}{c}\text { RODA } \\
3.0 \\
\end{array}$ \\
\hline Autenticación & 2 & 2 & 2 \\
\hline Diferentes permisos de acceso & 0 & 2 & 2 \\
\hline Registro de acciones de usuario & 0 & 2 & 2 \\
\hline Notificaciones & 0 & 2 & 2 \\
\hline Estadísticas & 0 & 2 & 2 \\
\hline SUBTOTAL & $2 / 10$ & $10 / 10$ & $10 / 10$ \\
\hline
\end{tabular}

Tabla 3. Funcionalidades relacionadas con la Administración del uso

Para garantizar la autenticidad e integridad de los documentos archivísticos es esencial que el software tenga la funcionalidad "diferentes permisos de acceso" y "registro de acciones de usuario", pues una define quién puede realizar diferentes acciones en el repositorio y en los documentos y la otra registra qué se ha realizado. No poseer estas funcionalidades por defecto, es decir, sin la necesidad de instalar otro software, es un punto débil que imposibilita la gestión de los documentos archivísticos en su totalidad.

Categoría Interfaz de usuario

\begin{tabular}{|l|c|c|c|}
\hline \multirow{2}{*}{\multicolumn{1}{|c|}{ Funcionalidades }} & \multicolumn{3}{c|}{ Puntuación } \\
\cline { 2 - 4 } & $\begin{array}{c}\text { Archivematica } \\
1.9 .1\end{array}$ & $\begin{array}{c}\text { DSpace } \\
6 . x\end{array}$ & $\begin{array}{c}\text { RODA } \\
3.0\end{array}$ \\
\hline Personalización & 1 & 2 & 1 \\
\hline Acceso multilingüe en la interfaz & 2 & 2 & 2 \\
\hline Administración multilingüe & 2 & 2 & 2 \\
\hline Adaptación para móvil & 0 & 2 & 0 \\
\hline \multicolumn{1}{|c|}{ SUBTOTAL } & $\mathbf{5 / 8}$ & $\mathbf{8 / 8}$ & $\mathbf{5 / 8}$ \\
\hline
\end{tabular}

Tabla 4. Funcionalidades relacionadas con la Interfaz de usuario 
De esta categoría se consideran como esenciales dos funcionalidades, acceso y la administración multilingües. Si el software no permite cambiar el idioma al portugués, dificultará o imposibilitará su uso.

Categoría Preservación digital

\begin{tabular}{|l|c|c|c|}
\hline \multirow{2}{*}{ Funcionalidades } & \multicolumn{2}{c|}{ Puntuación } \\
\cline { 2 - 4 } & $\begin{array}{c}\text { Archivematica } \\
1.9 .1\end{array}$ & $\begin{array}{c}\text { DSpace } \\
6 . x\end{array}$ & $\begin{array}{c}\text { RODA } \\
3.0\end{array}$ \\
\hline Modelo OAIS & 2 & 0 & 2 \\
\hline PREMIS & 2 & 2 & 2 \\
\hline METS & 2 & 2 & 2 \\
\hline EAD & 2 & 0 & 2 \\
\hline Dublin Core & 2 & 2 & 2 \\
\hline $\begin{array}{l}\text { Exportar a / Importar desde otro } \\
\text { formatos de metadatos }\end{array}$ & 2 & 2 & 2 \\
\hline Herramientas/servicios de conversión de formatos & 2 & 2 & 2 \\
\hline Validación e Identificación de formatos & 2 & 2 & 2 \\
\hline Respaldo/Copia de Seguridad & 2 & 2 & 2 \\
\hline \multicolumn{1}{|c|}{ SUBTOTAL } & $18 / 18$ & $14 / 18$ & $18 / 18$ \\
\hline
\end{tabular}

Tabla 5. Funcionalidades relacionadas con la Preservación digital

De las nueve funcionalidades seleccionadas para esta categoría, dos no son consideradas esenciales, Modelo OAIS y EAD, que ayudan en la preservación de los objetos digitales, pero no son esenciales. Dichas funcionalidades completan y amplían la preservación digital. Pero las demás garantizan la preservación de los objetos digitales.

Categoría: Interoperabilidad e Integración

\begin{tabular}{|l|c|c|c|}
\hline \multirow{2}{*}{ Funcionalidades } & \multicolumn{3}{c|}{ Puntuación } \\
\cline { 2 - 4 } & Archivematica & DSpace & RODA \\
& 1.9 .1 & $6 . x$ & 3.0 \\
\hline OIA-PMH & 2 & 2 & 2 \\
\hline Integración con otros sistemas & 2 & 2 & 2 \\
\hline Integración con los software evaluados & 2 & 2 & 0 \\
\hline SUBTOTAL & $6 / 6$ & $6 / 6$ & $6 / 4$ \\
\hline
\end{tabular}

Tabla 6. Funcionalidades relacionadas con la Interoperabilidad e Integración

La primera funcionalidad es esencial para cualquier biblioteca digital, y por lo tanto es la más importante para la BNDigital. En cuanto a la segunda, esta es imprescindible para el SEI, pues el software tendrá que estar integrado en él, ya que los procedimientos administrativos con trámites finalizados en el SEl serán transferidos al software para la gestión, preservación y acceso futuro. 
Resultados general por software

\begin{tabular}{|l|c|c|c|}
\hline \multicolumn{4}{|c|}{ Archivematica 1.9.1 } \\
\hline \multicolumn{1}{|c|}{ Categorías } & $\begin{array}{c}\text { Puntuación } \\
\text { obtenida }\end{array}$ & $\begin{array}{c}\text { Puntuación } \\
\text { máxima }\end{array}$ & $\%$ \\
\hline Infraestructura & 13 & 26 & $50 \%$ \\
\hline Gestión de contenido & 6 & 12 & $50 \%$ \\
\hline Administración del uso & 2 & 10 & $20 \%$ \\
\hline Interfaz de usuario & 5 & 8 & $63 \%$ \\
\hline Preservación digital TOTAL & 18 & 18 & $100 \%$ \\
\hline Interoperabilidad e Integración & 6 & 6 & $100 \%$ \\
\hline \multicolumn{1}{|c|}{ TOTA } & $\mathbf{8 0}$ & $\mathbf{6 2 \%}$ \\
\hline
\end{tabular}

Tabla 7. Resultado general Archivematica en cada categoría

A través del resultado obtenido en cada categoría se concluye que la preservación digital, interoperabilidad e integración son los puntos fuertes del software Archivematica y los débiles están en la infraestructura, gestión de contenido y administración del uso. Por los puntos fuertes se observa también que Archivematica no está indicado para ser utilizado solo, sino con otros sistemas. Y por sus puntos débiles se infiere que no atiende a las necesidades de la BNDigital.

\begin{tabular}{|l|c|c|c|}
\hline \multicolumn{4}{|c|}{ DSpace 6.x } \\
\hline \multicolumn{1}{|c|}{ Categorías } & $\begin{array}{c}\text { Puntuación } \\
\text { obtenida }\end{array}$ & $\begin{array}{c}\text { Puntuación } \\
\text { máxima }\end{array}$ & $\%$ \\
\hline Infraestructura & 24 & 26 & $92 \%$ \\
\hline Gestión de contenido & 12 & 12 & $100 \%$ \\
\hline Administración del uso & 10 & 10 & $100 \%$ \\
\hline Interfaz de usuario & 8 & 8 & $100 \%$ \\
\hline Preservación digital & 14 & 18 & $78 \%$ \\
\hline Interoperabilidad e Integración & 6 & 6 & $100 \%$ \\
\hline \multicolumn{1}{|c|}{ TOTAL } & 74 & 80 & $92 \%$ \\
\hline
\end{tabular}

Tabla 8. Resultado DSpace en cada categoría

De las seis categorías analizadas, DSpace cumple con el cien por cien en cuatro de ellas. A partir del buen resultado en la categoría infraestructura se puede inferir que su instalación es la menos costosa de los tres softwares evaluados y la madurez en el mercado le atribuye un grado mayor de sostenibilidad. Por cumplir con el $100 \%$ de las funcionalidades en las categorías de gestión de contenido, administración del uso e interfaz del usuario, se puede 
concluir que está indicado para la gestión de bibliotecas digitales. Su peor resultado fue en la categoría preservación digital, por no poseer funcionalidades complementarias.

\begin{tabular}{|l|c|c|c|}
\hline \multicolumn{4}{|c|}{ RODA 3.0 } \\
\hline \multicolumn{1}{|c|}{ Categorías } & $\begin{array}{c}\text { Puntuación } \\
\text { obtenida }\end{array}$ & $\begin{array}{c}\text { Puntuación } \\
\text { máxima }\end{array}$ & $\%$ \\
\hline Infraestructura & 15 & 26 & $58 \%$ \\
\hline Gestión de contenido & 10 & 12 & $84 \%$ \\
\hline Administración del uso & 10 & 10 & $100 \%$ \\
\hline Interfaz de usuario & 5 & 8 & $63 \%$ \\
\hline Preservación digital & 18 & 18 & $100 \%$ \\
\hline Interoperabilidad e Integración & 4 & 6 & $67 \%$ \\
\hline \multicolumn{1}{|c|}{ TOTAL } & $\mathbf{6 2}$ & $\mathbf{8 0}$ & $\mathbf{7 7 \%}$ \\
\hline
\end{tabular}

Tabla 9. Resultado RODA en cada categoría

Las categorías Administración del uso y Preservación digital son los puntos fuertes del software RODA, pues posee la totalidad de las funcionalidades. Presentó aun un buen resultado en las categorías Gestión de contenido e Interoperabilidad e integración, y en esta última tan solo no cuenta con una funcionalidad, que sería importante si dos de los tres softwares evaluados fueran considerados como los dos más adecuados.

En el gráfico 1 a continuación, se presenta el resultado general obtenido por cada software. DSpace fue el que presentó mejor resultado y cumple con el $92 \%$ de las funcionalidades seleccionadas para esta evaluación. En segundo lugar está RODA, con un nivel de satisfacción del $77 \%$ y en último lugar está Archivematica, con un 62\%. En el apartado siguiente se presenta un análisis de la puntuación atribuida a cada software por categoría. 


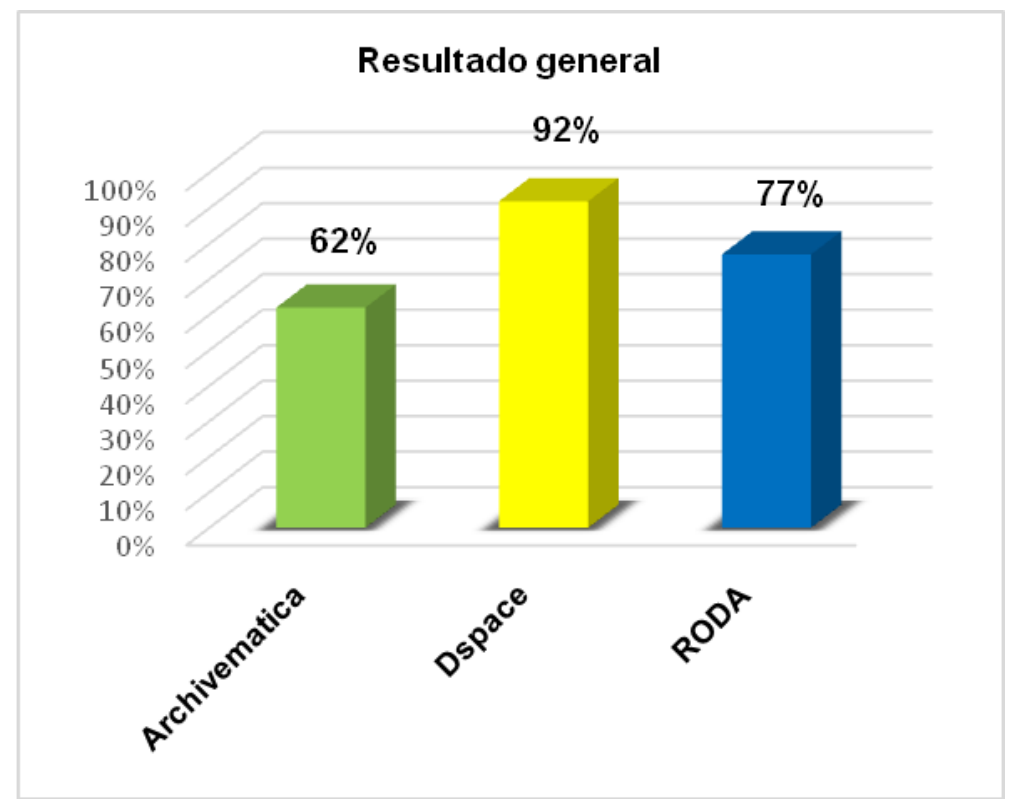

Gráfico 1. Resultado general

\section{Conclusiones}

La presente investigación evaluó los softwares Archivematica, DSpace y RODA, con el fin de identificar el más adecuado para la gestión y preservación de los materiales bibliográficos y de los documentos archivísticos de la Biblioteca Nacional de Brasil. La evaluación demostró que Archivematica, uno de los softwares evaluados, no es un software para repositorio digital, sino un sistema de preservación digital, es decir, una solución tecnológica para un repositorio digital. La investigación llegó a esta conclusión porque, en este trabajo, se consideró repositorio digital el medio de almacenamiento que preserva, difunde y facilita el acceso a los objetos digitales que almacena. De acuerdo con el resultado de la evaluación, Archivematica no provee acceso a los objetos digitales, así que para acceder al contenido preservado por el programa, es necesario la instalación e integración con otro software. El software tampoco posee funcionalidades importantes para la gestión de contenido como: informes estadísticos, gestión de diferentes permisos de acceso, notificaciones de las acciones que los usuarios realizan en el sistema y búsqueda por texto integral. Al no poseer estas funcionalidades no se caracteriza como un software para repositorio digital y no es el más adecuado para la gestión de materiales bibliográficos de una biblioteca digital. Sin embargo, la investigación también constató que Archivematica es el software para preservación digital más reconocido en el mercado, pues fue desarrollado con la colaboración de la UNESCO, a través del Memory of the World's - Subcommittee on Technology.

El software RODA cumple con el 77\% de las funcionalidades seleccionadas para esta 
evaluación. Sin embargo, es un software de preservación OAIS y no una plataforma de publicación. El uso más común de RODA es permitir que un archivo o biblioteca sea accesible solo para los usuarios de dentro de una institución, no obstante es posible hacer públicos los objetos digitales. RODA posee algunas funcionalidades en común con Archivematica, porque ambos están basados en el modelo OAIS. La diferencia es que RODA es un software que contiene algunas funcionalidades ya integradas en la aplicación como el acceso, no habiendo necesidad de instalación e integración con otro software, y también dispone de funcionalidades de gestión. El uso del software está disponible en el mercado desde 2009, pero todavía no está ampliamente difundido, existen pocas empresas que lo utilizan, su comunidad de soporte contiene pocos colaboradores.

La bibliografía revisada ya calificaba DSpace como el software más adecuado para implantación en bibliotecas digitales, es decir, para gestionar materiales bibliográficos y los datos acerca del porcentaje de instalaciones registradas en el mundo y los tipos de instituciones que utilizan el software corroboran esta calificación. Planteaba dudas si DSpace también era adecuado para la gestión de documentos archivísticos y para la preservación digital. Al cumplir con el cien por cien de las categorías gestión de contenido y administración del uso, se comprobó que es el software más adecuado para la gestión de objetos y documentos digitales.

En la categoría preservación digital, el porcentaje de cumplimiento de DSpace fue inferior a la de los otros softwares evaluados, pero de acuerdo con los estudios previos a esta investigación DSpace, así como Archivematica y RODA, tan solo no garantizan la preservación de los objetos digitales, pues los softwares de repositorio son solamente una parte del amplio panorama que constituye la preservación digital. Aunque Archivematica y RODA cumplen a más funcionalidades que DSpace en la categoría preservación digital, ellos no son una solución aislada que garantice el acceso a los objetos digitales a largo plazo. Gran parte de los atractivos de dichos softwares proviene del hecho de que integran muchas otras herramientas de código abierto, que realizan funciones específicas y complementarias para el complejo y amplio objetivo que es la preservación digital a largo plazo.

DSpace, con la más grande comunidad de desarrolladores y administradores del mundo, está evolucionado desde repositorios institucionales hasta repositorio de documentos administrativos. Esto viene avalado por la creciente cantidad de estudios de casos y publicaciones del uso de DSpace como herramienta para repositorio de documentos administrativos y también su utilización por instituciones como el Departamento 
Administrativo de Ciencia, Tecnología e Innovación ${ }^{1}$ de Colombia y el Development Bank of Latin America², de Estados Unidos.

Por la explicación en los párrafos anteriores y por el resultado general de la evaluación realizada en esta investigación, DSpace es el software más adecuado para la gestión y preservación de los materiales bibliográficos y de los documentos archivísticos de la Biblioteca Nacional de Brasil, atendiendo al 92\% de las funcionalidades consideradas como las más importantes y relevantes para la gestión y preservación de objetos digitales. Aunque algunos autores consideran que DSpace no contempla la preservación digital y el almacenamiento de documentos archivísticos digitales, en su totalidad el software es el que mejor atiende a las necesidades de la Biblioteca Nacional de Brasil, entre todos los softwares evaluados, ya que el programa más adecuado debe atender a las necesidades de la BNDigital, que tiene como propósito preservar los materiales bibliográficos para proveer el acceso a ellos.

La cantidad de funcionalidades que DSpace posee para preservación de los documentos archivísticos digitales producidos por el SEI es mucho más que las ofrecidas por Archivematica y RODA para la gestión de la BNDigital. La numerosa y amplia comunidad de usuarios, desarrolladores y administradores que DSpace posee es considerada por la comunidad de preservación digital como un indicador confiable de la longevidad del software, y esto es una característica esencial para la preservación a largo plazo.

\section{Referencias}

ÁLVAREZ WONG, B. Los repositorios digitales para la conservación. Un acercamiento a la preservación digital a largo plazo. Ciencias de la Información, v. 48; n.2, p. 15-22, 2017. Recuperado de: http://www.redalyc.org/articulo.oa?id=181454540003

ALVITE, M.; RODRÍGUEZ, B. Evaluación de interfaces de OPACs implementadas con UNICORN en las Universidades madrileñas. III Jornadas españolas de bibliotecas digitales. Madrid: Universidad Politécnica de Madrid, 2002. Recuperado de: https://buleria.unileon.es/bitstream/handle/10612/3068/UNICORN\%20en\%20universidades\% 20madrile\%C3\%B1as.pdf?sequence $=4$

${ }^{1}$ http://repositorio.colciencias.gov.co/

${ }^{2}$ http://www.scioteca.caf.com/ 
BANKIER J. G.; Gleason K. Institutional Repository Software Comparison.2014. Recuperado de: https://unesdoc.unesco.org/ark:/48223/pf0000227115

BRASIL. CONSELHO NACIONAL DE ARQUIVOS. Diretrizes para a implementação de repositórios digitais confiáveis de documentos arquivísticos. Rio de Janeiro: Arquivo Nacional, 2014. Recuperado de: http://conarq.arquivonacional.gov.br/images/publicacoes textos/diretrizes rdc arq.pdf

BRASIL. CONSELHO NACIONAL DE ARQUIVOS. e-ARQ Brasil: Modelo de Requisitos para Sistemas Informatizados de Gestão Arquivística de Documentos. Rio de Janeiro: Arquivo Nacional, 2011.2 Recuperado de: http://conarq.arquivonacional.gov.br/images/publicacoes textos/earqbrasil model requisitos 2009.pdf

BRASIL. Decreto 8.297/2014, de 15 de agosto de 2014, aprova o Estatuto e o Quadro de Demonstrativo dos Cargos em Comissão da Fundação Biblioteca Nacional. Diário Oficial da República Federativa do Brasil, 2014.2 Recuperado de: http://www.planalto.gov.br/ccivil 03/ Ato2011-2014/2014/Decreto/D8297.htm

BRASIL. Decreto 8.539/2015, de 8 de outubro de 2015, dispõe sobre o uso do meio eletrônico para realização do processo administrativo. Diário Oficial da República Federativa do Brasil,2015. Recuperado de: http://www.planalto.gov.br/ccivil 03/ Ato20152018/2015/Decreto/D8539.htm

HEMINGER, A.; ROBERTSON, S. The Digital Rosetta Stone: A Model for Maintaining Long-term Access to Static Digital Documents. Communications of the Association for Information Systems, 3, 2000.

MASREK M.N. HAKIMJAVADI, H. Evaluation of Three Open Source Software in Terms of Managing Repositories of Electronic Theses and Dissertations: A Comparison Study. Journal of Basic and Applied Scientific Research, v. 2; n. 11, 2012. Recuperado de: https://pdfs.semanticscholar.org/4f20/fa7f78e82ed10b57ec0eb6e54dd29e23d0f1.pdf? ga=2. 31173943.928927258.1563125175-1286103302.1560078805

MATEUS, J. C. O Governo Eletrónico, a sua aposta em Portugal e a importância das Tecnologias de Comunicação para a sua estratégia. Revista de Estudos Politécnicos, v. 4; n. 9, p. 31-52, 2008. Recuperado de: http://www.scielo.mec.pt/pdf/tek/n9/n9a02.pdf

POLANCO-CORTÉS, J. Repositorios digitales: Definición y pautas para su creación, 2014. Recuperado de: https://ucrindex.ucr.ac.cr/docs/repositorios-digitales-definicion-y-pautaspara-su-creacion.pdf

ROSA, C. A., CRAVEIRO, O.; DOMINGUES, P. Open source software for digital preservation repositories: A survey. International Journal of Computer Science and Engineering Survey (IJCSES), v. 8; n. 3, 2017. Recuperado de: https://arxiv.org/abs/1707.06336

TEXIER, J. Repositorio de Documentos Administrativos para la Universidad Nacional Experimental del Táchira (REDAUNET). 2013. Recuperado en: http://eprints.rclis.org/20813/ 